\title{
Key Building Block Optimization for High- Performance Transceivers: Multimode Interferometers from Conventional to Sub- Wavelength Regime
}

\author{
Yunlong Zhang ${ }^{*}$ Marc Schneider, Djorn Karnick, Lars Eisenblätter, Thomas \\ Kühner, Marc Weber \\ Karlsruhe Institute of Technology, Institute for Data Processing and Electronics \\ Hermann-von-Helmholtz-Platz 1, 76344 Eggenstein-Leopoldshafen, Germany \\ E-mail: zhang.yunlong@partner.kit.edu, djorn.karnick@kit.edu, \\ marc.schneider@kit.edu, lars.eisenblaetter@kit.edu, \\ thomas.kuehner@kit.edu, marc.weber@kit.edu
}

\begin{abstract}
Multimode interferometers (MMIs) are key components for high-bandwidth transceivers in upgrading the data transmission of future detector systems. We present two conventional highperformance MMIs fabricated on a $250 \mathrm{~nm}$ SOI platform with different power splitting ratios which operate as 50\%:50\% power splitter for Mach-Zehnder modulators and as 86\%:14\% power splitter for loop control in future transceiver designs, respectively. Besides, we present the novel MMIs based on sub-wavelength gratings. By engineering the refractive index of relevant parts, more compact 50\%:50\% MMIs and multi-port, low phase error MMIs become feasible.
\end{abstract}

Topical Workshop on Electronics for Particle Physics (TWEPP2019)

2- 6 September 2019

Santiago de Compostela, Spain

\footnotetext{
${ }^{*}$ Speaker
} 


\section{Introduction}

The Talbot effect was first observed in 1836 [1]. It implies that a monochromatic optical planar wave hitting upon a periodic diffraction grating, will create repeated grating images at a fixed distance L, away from the diffraction grating. The regular distance $\mathrm{L}$ is called the Talbot length. After lots of attempts of analysis and derivation [2]-[6], the theory and understanding of this phenomenon became mature. The fundamental idea of multimode interferometers (MMIs) is to induce a confined Talbot effect [7]-[8], and this concept was tested by Ulrich et al. [9]. Since then, MMIs received significant attention and were widely applied, especially in silicon photonics integration, due to their compactness and broadband characteristics [10] [12].

With the development of fabrication technology, researchers tried to improve the performance of MMI further with sub-wavelength gratings. A sub-wavelength grating is a periodic structure at a scale smaller than the wavelength of the light propagating through it. Thereby diffraction effects are suppressed and the corresponding waveguide can be regarded as a homogeneous waveguide. Literature [13] shows that the performance of MMIs deteriorates dramatically, when they are fabricated on a high-index contrast platform, for instance silicon-on -insulator (SOI). Under these circumstances, utilizing sub-wavelength structures is very attractive [14]-[15].

In this paper, we present the design of two different conventional MMIs with different splitting ratios, rigorous simulation results from COMSOL Multiphysics and measurement results of the fabricated devices. In addition, the sub-wavelength MMIs were studied and the corresponding simulation results are presented as well.

\section{Conventional and sub-wavelength MMIs}

\subsection{MMI fundamentals}

The design of conventional MMIs usually starts from the calculation of propagation constants, which are calculated by (1) [10], where $\mathrm{k}$ is the free space wavenumber, $\mathrm{n}$ is the refractive index of the waveguide core, $\mathrm{W}$ is the width of MMI and $\mathrm{m}$ is the mode order. The required length of the device is dominantly determined by the beat length $L_{\pi}$ (2) [16], therefore the first and second order propagation constants are rather important. It is worth mentioning that, for high-quality imaging, the propagation constants are required to satisfy the quadratic relation in (3) [16].

(1) $\beta_{m}=k n \sqrt{1-\left(\frac{m \pi}{W k n}\right)^{2}}$

(2) $L_{\pi}(\lambda)=\frac{\pi}{\beta_{1}(\lambda)-\beta_{2}(\lambda)}$

(3) $\beta_{m}=\beta_{1}-\left(m^{2}-1\right) \pi /\left(3 L_{\pi}\right)$

Taking the number of ports, their relative positions and the length of the multimode region into account, the number of images and their optical intensity ratio can be designed and selected accordingly. There have already been abundant publications about this analysis and calculation [10] [16] so that this will not be the focus of this paper. 


\subsection{Two conventional MMIs}

3-dB (50\%:50\% power splitting ratio) MMIs are widely used as power splitter and power combiner. In our design, we set the width of the MMI to $4.5 \mu \mathrm{m}$ and swept the length of the MMI to optimize the design via numerical simulation. The optimal length of the multimode region is $24.5 \mu \mathrm{m}$. In the same way, we optimized the geometry of an 86\%:14\% MMI, resulting in a geometry of $2.8 \mu \mathrm{m} \times 14 \mu \mathrm{m}$. Fig. 1 and Fig. 2 show numerical simulations of two MMIs at $1550 \mathrm{~nm}$ in COMSOL Multiphysics.

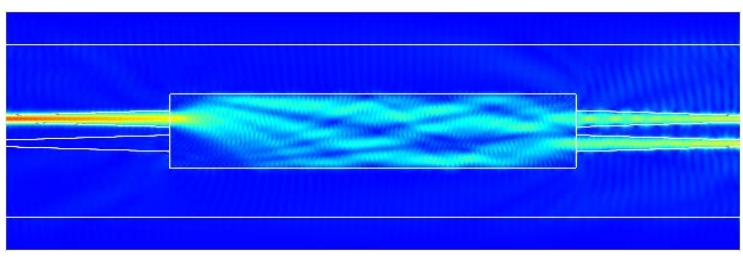

Fig. 1 50\%:50\% MMI simulation

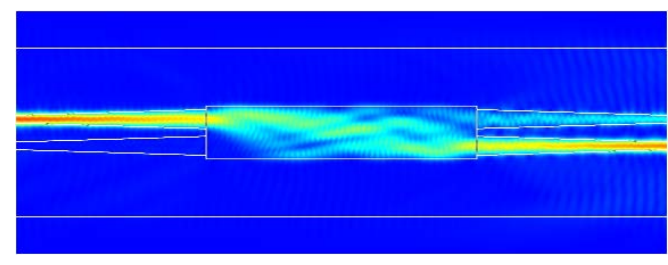

Fig. 2 86\%:14\% MMI simulation

We fabricated the above two devices on a $250 \mathrm{~nm}$ SOI platform at IMS Chips (Institut für Mikroelektronik Stuttgart, Germany) and characterized them. The measurement and simulation results are illustrated in Fig. 3 a) and b), respectively. It can be seen that theory and experiment are in good agreement with nearly negligible differences. The total insertion loss of both MMIs is below $0.5 \mathrm{~dB}$. Additionally, their splitting ratio remains rather stable throughout the $\mathrm{C}$ band.

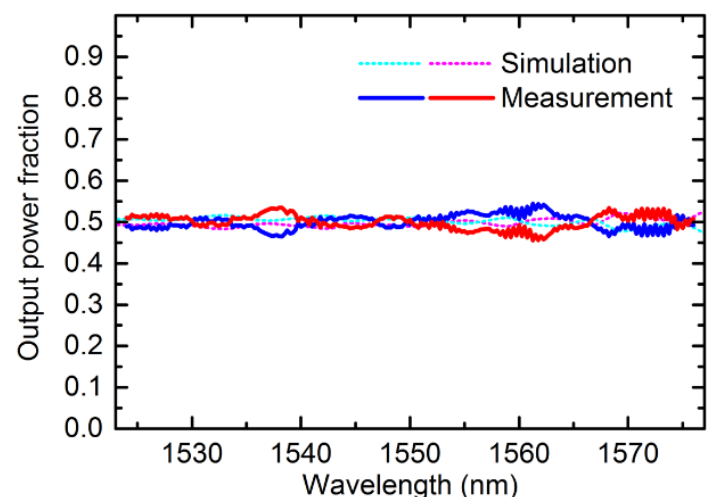

a)

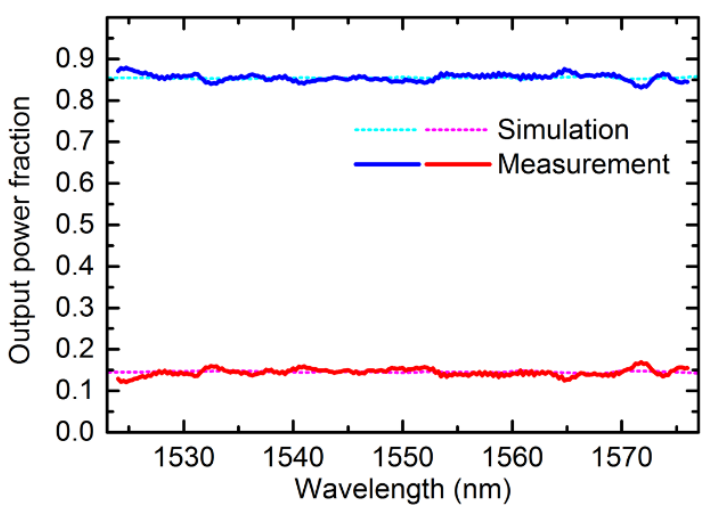

b)

Fig. 3 Output power fraction versus incident wavelength of the a) 50\%:50\% MMI and b) 86\%:14\% MMI.

\subsection{A sub-wavelength 50\%:50\% 2×2 MMI}

Using the identical width and position of the I/O ports as the device simulated in Fig. 2, we engineered the equivalent refractive index of the middle part of the multimode region, which modifies the profile of even modes. Therefore, the phase difference of the first two order modes will be altered accordingly. The simulation shows required length of 50\%:50\% MMIs is $14 \mu \mathrm{m}$.

One effective way of engineering the refractive index is using sub-wavelength gratings. To start, Rytov's formulae (4) and (5) [14] are important where $n_{\downarrow}$ and $n_{\perp}$ are the directions parallel and perpendicular to the interfaces between the alternating layers, respectively. As Fig. 4 shows, a is the length of the grating and $\Lambda$ is the pitch of the periodic structure. Optimized parameters are $L_{\text {MMI }}=14 \mu \mathrm{m}$ (length of the MMI), a $=60 \mathrm{~nm}$ and $\Lambda=250 \mathrm{~nm}$. The final structure can be seen in Fig. 5 where a row of holes runs horizontally through the center of the MMI, 
representing the sub-wavelength grating, where the silicon layer is etched down to the substrate. An inset picture zoomed into the sub-wavelength gratings is inserted to Fig. 5 as well to help understand the structure. The simulated insertion loss of this device within the $\mathrm{C}$ band is lower than $0.3 \mathrm{~dB}$ and thus marginally better than the value of the conventional $50 \%: 50 \% \mathrm{MMI}$ presented in Fig. 1, while the on-chip footprint is $38 \%$ smaller.

(4) $n_{\|}^{2}=\frac{a}{\Lambda} n_{1}^{2}+\left(1-\frac{a}{\Lambda}\right) n_{2}^{2}$

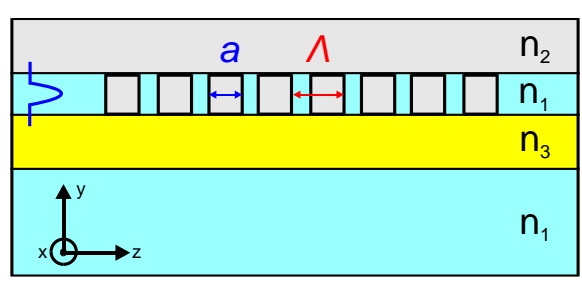

Fig. 4 Sub-wavelength structures from the side view
(5) $\frac{1}{n_{\perp}{ }^{2}}=\frac{a}{\Lambda} \frac{1}{n_{1}^{2}}+\left(1-\frac{a}{\Lambda}\right) \frac{1}{n_{2}^{2}}$

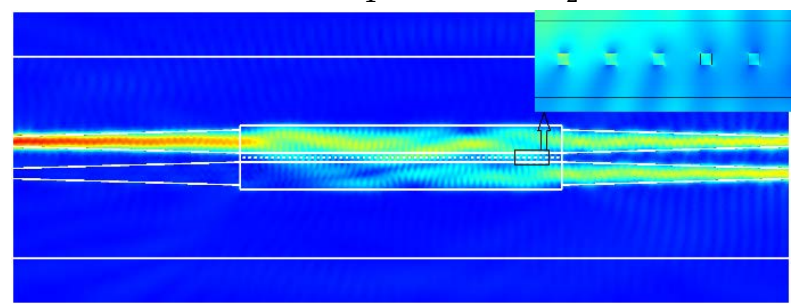

Fig. 5 Simulation of 3-dB sub-wavelength MMI

\subsection{A sub-wavelength $4 \times 4$ MMI}

Based on the previous investigations, we extended the design to multi-port MMIs and present a $4 \times 4 \mathrm{MMI}$ in this section. According to [13], the modal phase error increases with the number of modes and strongly depends on the refractive index contrast. To improve the performance of $n \times n$ MMIs, we engineered the refractive index of the side region next to the multimode region to lower the refractive index contrast. Fig. 6 shows the simulation of the proposed $4 \times 4$ MMI in COMSOL Multiphysics. The footprint of this sub-wavelength MMI is $7.2 \mu \mathrm{m} \times 106 \mu \mathrm{m}$, slightly larger than the conventional MMI $(7.2 \mu \mathrm{m} \times 96 \mu \mathrm{m})$, and its side region has an equivalent refractive index of 2.64 at $1550 \mathrm{~nm}$. Both the conventional and the subwavelength 4×4 MMI have an insertion loss around $0.4 \mathrm{~dB}$ at $1550 \mathrm{~nm}$, whereas the maximum phase error decreases significantly from $10.3^{\circ}$ to $3.1^{\circ}$ for the sub-wavelength MMI.

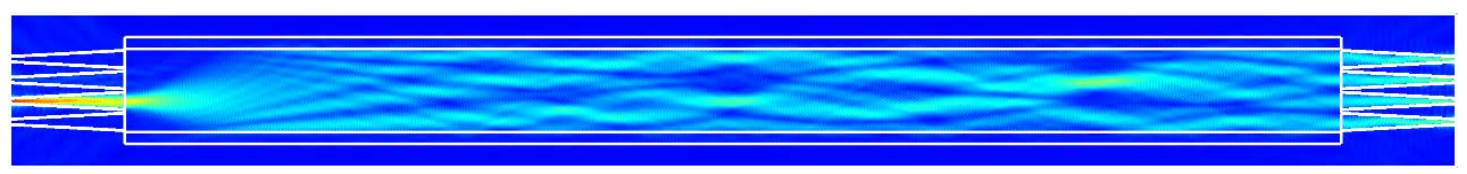

Fig. 6 Simulation of a $4 \times 4$ MMI with side regions of engineered refractive index

\section{Conclusion}

A basic design process of $n \times n$ MMIs is presented in this paper. Two conventional MMIs with different power splitting ratios were simulated and fabricated on a $250 \mathrm{~nm}$ SOI platform: 50\%:50\% and the 86\%:14\%, respectively. Our simulations are validated through their excellent agreement with the measurement results. Due to their compact structure and low losses, they will be key components for the modulators and loop control in our integrated transceivers designs. By engineering the refractive index of the relevant regions, the 50\%:50\% 2×2 MMI can be made even smaller compared with conventional ones, and the modal phase error of $4 \times 4$ MMIs can be decreased dramatically by a factor of 3. The study in this paper will contribute to 
the optimization of our high-bandwidth transmitter [12] design to upgrade detector readout systems.

\section{Acknowledgments}

This research is supported by the program Matter and Technology of the Helmholtz Association, Germany and the China Scholarship Council (CSC), China.

\section{References}

[1] H. F. Talbot, Facts relating to optical science, Philos. Mag. 9(56), 403-405 (1836).

[2] L. Rayleigh, On Copying Diffraction Gratings, and some Phenomena Connected Therewith, Philos. Mag. 11(67), 196-205 (1881).

[3] J. M. Cowley and A. F. Moodie, Fourier Images, Proc. Phys. Soc. B 70, 486 (1957).

[4] J. T. Winthrop, and c. R. Worthington, Theory of Fresnel Images. I. Plane Periodic Objects in Monochromatic Light, J. Opt. Soc. Am. 55, 373-381 (1965).

[5] M. V. Berry, et al. Quantum Carpets, Carpets of Light, Phys. World 16 (6), (2001).

[6] J. Wen et al. The Talbot effect: recent advances in classical optics, nonlinear optics, and quantum optics, Adv. Opt. Photon. 5 (1), 83-130 (2013).

[7] L. A. Rivlin and V. S. Shul'dyaev, Multimode waveguides for coherent light, Zzv. VUZ Radiofizika 11(4), 572-578 (1968).

[8] O. Bryngdahl, Image formation using self-imaging techniques, J. Opt. Soc. Am. 63(4), 416-419 (1973).

[9] R. Ulrich and G. Ankele, Self-imaging in homogeneous planar optical waveguides, Appl. Phys. Lett. 27(6), 337-339 (1975).

[10] Cooney, Kieran, and Frank H. Peters. Analysis of multimode interferometers, Optics express 24.20 (2016): 22481-22515.

[11] Y. Zhang, et al. Concept, design and verification of components for an integrated on-detector silicon photonic multichannel transmitter, PoS (2019): 057.

[12] Y. Zhang, et al. Key building blocks of a silicon photonic integrated transmitter for future detector instrumentation, Journal of Instrumentation 14.08 (2019): P08021.

[13] J. Z. Huang, et al. A new design approach to large input/output number multimode interference couplers and its application to low-crosstalk WDM routers, IEEE Photonics Technology Letters 10.9 (1998): 1292-1294.

[14] R. Halir, et al. Waveguide sub-wavelength structures: a review of principles and applications, Laser \& Photonics Reviews 9.1 (2015): 25-49.

[15] R. Halir, et al. Subwavelength-grating metamaterial structures for silicon photonic devices, Proceedings of the IEEE 106.12 (2018): 2144-2157.

[16] M. Bachmann, et al. General self-imaging properties in $N \times N$ multimode interference couplers including phase relations, Applied optics 33.18 (1994): 3905-3911. 